\title{
Electrochemical measurement of dopamine release and uptake in zebrafish following treatment with carboplatin
}

\author{
Dr. Thomas M. Field, \\ Department of Chemistry, University of Kansas, 1251 Wescoe Hall Drive, Lawrence, KS 66045 \\ USA \\ Dr. Mimi Shin, \\ Department of Chemistry, University of Kansas, 1251 Wescoe Hall Drive, Lawrence, KS 66045 \\ USA \\ Chase S. Stucky, \\ Department of Chemistry, University of Kansas, 1251 Wescoe Hall Drive, Lawrence, KS 66045 \\ USA \\ Joseph Loomis, and \\ Department of Chemistry, University of Kansas, 1251 Wescoe Hall Drive, Lawrence, KS 66045 \\ USA \\ Prof. Michael A. Johnson \\ Department of Chemistry and Graduate Program in Neuroscience, University of Kansas, 1251 \\ Wescoe Hall Drive, Lawrence, KS 66045 USA
}

\section{Abstract}

Post chemotherapy cognitive impairment, also known as 'chemobrain,' is a neurological condition in which cognitive function is impaired as a result of cancer chemotherapy treatment. In this work, we used fast-scan cyclic voltammetry (FSCV) to measure electrically evoked dopamine release and uptake in whole brain preparations from zebrafish that have been treated with carboplatin, an agent associated with chemobrain. We administered carboplatin by addition to the fish's tank water or their food. One week of treatment with $100 \mu \mathrm{M}$ carboplatin in the water was needed to significantly impair dopamine release ( $40 \%$ of control); however, only one day of treatment through the zebrafish's food was needed to cause a similar impairment. Atomic absorption spectroscopy measurements suggested that administration through food resulted in higher initial levels of carboplatin compared to water administration, but water administration resulted in an increase over time. Uptake, determined by modeling stimulated release plots, was unaffected. These results are consistent with our previous findings of diminished neurotransmitter release in rats and support a role for zebrafish in chemobrain-related studies.

\section{Graphical Abstract}

Correspondence to: Michael A. Johnson. Note: TMF and MS contributed equally 
The administration of chemotherapeutic agents for the treatment of cancers often results in cognitive dysfunction, a condition known as chemobrain. Fast-scan cyclic voltammetry measurements in zebrafish treated with carboplatin, a commonly employed chemotherapy agent, reveal a decrease in dopamine release that is dependent upon the amount of carboplatin absorbed into the fishes' system. Image credit: David Dohnal/Shutterstock.com

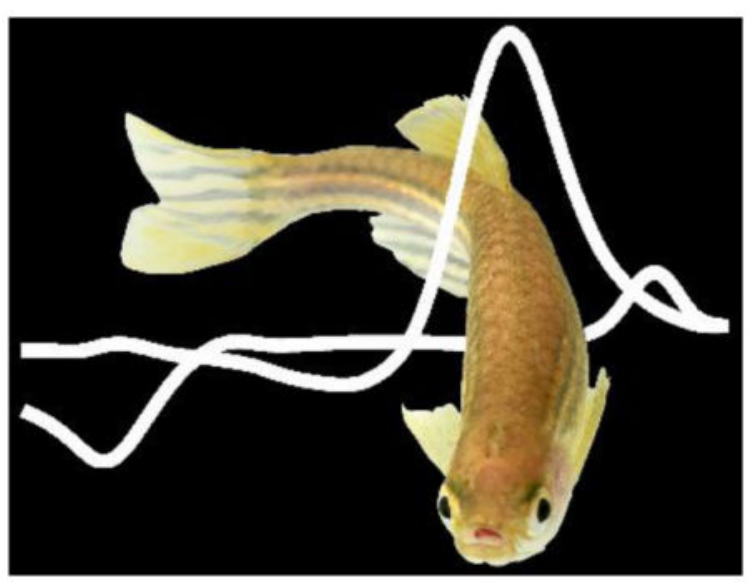

\section{Keywords}

voltammetry; dopamine; electrochemical; zebrafish; brain

\section{Introduction}

Post chemotherapy cognitive impairment, also known as 'chemobrain,' is a neurological condition characterized by a decrease in higher level cognitive and executive function after the conclusion of the treatment regime ${ }^{[1]}$. Although the underlying causes of chemobrain are not well understood, mechanisms that have been proposed include chemotherapy-induced DNA damage, disruption of vascular blood flow in the brain, inflammatory responses to reactive oxygen species, and impairment of neurotransmitter signals ${ }^{[2]}$. Recent in vivo studies of chemobrain have largely relied upon the use of rats and mice. For example, in our own work, we used fast-scan cyclic voltammetry at carbon-fiber microelectrodes (FSCV) to show that dopamine and serotonin release and cognitive performance were impaired in rats treated with carboplatin ${ }^{[3]}$, a chemotherapeutic agent commonly used in the treatment of cancers of the head, neck, breast, and lung ${ }^{[4]}$.

The zebrafish (Danio rerio), a teleost originally used to study development, has recently emerged as a useful model of neurochemical signaling ${ }^{[5]}$ and toxicology ${ }^{[6]}$. As a model of neuronal function, zebrafish represent an ideal compromise between brain complexity and small dimensions. The total number of cells in the adult brain has been estimated at 8 to 13 million ${ }^{[7]}$ while the dimensions are in the low- to sub-mm range ${ }^{[8]}$. This small size allows the whole brain to be kept alive ex vivo by simple perfusion ${ }^{[5 a]}$. Zebrafish are also useful for toxicological evaluation studies because dosing often requires only adding agents to the water or food, and behavioral and neurochemical analyses can be carried out with greater throughput and lower cost ${ }^{[9]}$ compared to larger organisms, such as rats. 
Recently, our group and others demonstrated the feasibility of using zebrafish whole brain as a preparation for measuring neurotransmitter release and uptake with $\mathrm{FSCV}^{[5]}$. In this work, we extend the application of this preparation toward the evaluation of carboplatin on dopamine release and uptake properties. We administered carboplatin to zebrafish by addition of this agent to either their water habitat or their food. Next, we quantified dopamine release and uptake in the whole brain ex vivo. This study revealed a sharp decrease in dopamine release after carboplatin treatment with food, while treatment of the water had a less significant effect. Thus, there was a strong influence of dosing regimen and exposure time on dopamine release. This work suggests that, similar to rats, zebrafish might be an effective model of neurotransmitter release impairment in chemobrain.

\section{Results and Discussion Carboplatin water treatment}

Recently, our group showed that carboplatin treatment caused a marked attenuation of dopamine release and cognitive decline in rats ${ }^{[3]}$. With these results in mind, we treated zebrafish with carboplatin and carried out dopamine release and uptake measurements. In the initial experiments, zebrafish were continuously treated through direct addition of either $100 \mu \mathrm{M}$ carboplatin or an equal volume of biological saline into their habitat water and neurochemical measurements were taken from ex vivo whole brain preparations after 1, 4, or 7 days.

As shown in Figures 1A and B, data from multiple fish were analyzed and a significant decrease in dopamine release was found after 7 days of treatment (two-way ANOVA, overall drug effect, $\mathrm{p}<0.05$; Sidak's multiple comparison test: 1-day $0.60 \pm 0.14 \mu \mathrm{M}$ control, 0.35 $\pm 0.09 \mu \mathrm{M}$ treated, $\mathrm{p}=0.017$; 4-day $0.41 \pm 0.07 \mu \mathrm{M}$ control, $0.29 \pm 0.09 \mu \mathrm{M}$ treated, $\mathrm{p}=$ 0.70; 7-day $0.63 \pm 0.06 \mu \mathrm{M}$ control, $0.27 \pm 0.04 \mu \mathrm{M}$ treated, $\mathrm{p}<0.05, \mathrm{n}=5$ brains per group). This decrease is consistent with previously published results in which rats were treated with carboplatin over the course of four weeks and dopamine release was measured in brain slices ${ }^{[3]}$.

\section{Carboplatin food treatment}

Although treatment through the water habitat resulted in an overall drug effect and a measurable decrease in dopamine release by day 7 , we sought to determine a treatment method that would yield more robust results.

While it has been shown that fish gills can accumulate unwanted metals in contaminated water, food treatment has been found in laboratory experiments to be the more important pathway for the delivery of molecules ${ }^{[10],[11]}$; therfore we administered the drug through their food. This was done for two reasons: 1) administering carboplatin through food retains the ease of use of water treatment and 2) carboplatin is known to cross the intestines in rodent models ${ }^{[12]}$.

During this treatment, we gave fish carboplatin infused brine shrimp once per day for 1,4 , or 7 days, after which dopamine measurements were made from whole zebrafish brain ex vivo. We estimated from atomic absorption (AA) spectroscopy measurements that the amount of 
carboplatin given per dose per fish was $236 \pm 44 \mu \mathrm{g}$, or about $472 \pm 88 \mathrm{mg}-\mathrm{kg}^{-1}$, assuming fish equally consumed the shrimp. As shown in Figure 2, the representative data reveal a significant attenuation in the dopamine release after just one day of food treatment. This attenuation appears to remain constant even after further treatments. Analysis of multiple fish show a dose dependent change in dopamine release that was significant compared to the control (two-way ANOVA, overall drug effect, $\mathrm{p}<0.0001$; Sidak's multiple comparison test: 1-day $0.47 \pm 0.07 \mu \mathrm{M}$ control, $0.21 \pm 0.05 \mu \mathrm{M}$, treated $\mathrm{p}<0.05$; 4-day $0.53 \pm 0.06 \mu \mathrm{M}$ control, $0.23 \pm 0.04 \mu \mathrm{M}$ treated, $\mathrm{p}<0.01$; 7-day $0.52 \pm 0.09 \mu \mathrm{M}$ control, $0.15 \pm 0.04 \mu \mathrm{M}$ treated, $\mathrm{p}<0.01 ; \mathrm{n}=5$ brains per group).

These findings of decreased dopamine release suggest that the effect of carboplatin on neurotransmitter release in zebrafish is similar to that in rodents. However, carboplatin delivered orally was more effective than when delivered by treating the habitat water. This observed difference in release may be related to the amount of intact drug absorbed into the animal's system.

To determine relative amounts of carboplatin absorbed, we treated zebrafish for 1 and 7 days with both methods and then used graphite furnace AA spectroscopy to quantify carboplatin in whole fish homogenates. The results, shown in Figure 3, reveal that, under the conditions used, the route of administration significantly affected how much carboplatin is absorbed into the fishes' body (two-way ANOVA, overall treatment effect, $\mathrm{p}<0.0001, \mathrm{n}=3$ to 4 fish per group). Also, amounts of carboplatin absorbed were significantly different between the two treatments at day 1 , but not day 7 ( $p<0.0001$, Sidak's multiple comparison test). Moreover, water treatment resulted in a substantial increase in content over 7 days $(\mathrm{p}<$ $0.0005)$ while food treatment resulted in a decrease in content $(\mathrm{p}<0.01)$.

Collectively, these results indicate that route of administration and duration of exposure when treating the water is important in determining how much carboplatin is absorbed by the fish. In the case of administration through water, it appears that carboplatin absorption into the tissues occurs over time, while administration through food causes a rapid increase at day 1 and decrease by day 7 . In the case of water administration, we speculate that it takes time for carboplatin to build up to a threshold level that could influence dopamine release. On the other hand, decreased carboplatin through food administration might be the result of increased clearance of the drug. The high initial levels of carboplatin provided by food administration likely supply ample chemotherapeutic agent for altering dopamine release. However, more work is required to understand the underlying mechanisms that impair dopamine release.

During food treatment, since the fish are ingesting the drug instead of absorbing it through the skin, and it is known that carboplatin can cross the small intestine of rats ${ }^{[12]}$, they are more likely to receive the entire intended dose leading to the observed decrease in release. This concept is supported by the fact that dopamine release impairment was not observed until after one week, whereas in the shrimp-treated fish dopamine release was impaired after one day. Additionally, shrimp-treated fish stored more carboplatin into their tissues than water-treated fish, indicating a more efficient dosing mechanism via ingestion. We were unable to detect platinum in the zebrafish brain with graphite furnace atomic absorption 
spectroscopy (AA; data not shown). It is possible, nevertheless, that trace amounts of carboplatin, undetectable by this particular analytical method, are still present. However, we also note that entry into the brain may not be necessary to affect brain viability. For example, doxorubicin, another commonly used chemotherapy agent, causes chemobrain even though it does not cross the blood brain barrier ${ }^{[13]}$. Moving forward, it will be important to determine how chemotherapy treatment impacts cognitive function in zebrafish and how cognitive changes correlate with neuronal function.

\section{Kinetics of dopamine uptake}

In the preceding section we showed that chemotherapy treatment impaired electrically evoked dopamine. With this result in mind, we wanted to determine if uptake was also affected by modelling the $1^{\text {st }}$ order rate constant of uptake $(\mathrm{k})$. Understanding uptake is important because it influences peak extracellular dopamine levels and the length of time dopamine is available to activate signaling pathways. The blue line in Figure 4 is a fit of the data obtained using the equation $\mathrm{A}_{\mathrm{t}}=\mathrm{A}_{\max } \mathrm{e}^{-\mathrm{kt}}$ from the point of maximum signal to $80 \%$ decay of the signal. $A_{\max }$ was held at the experimentally determined maximum for release and $\mathrm{k}$ was allowed to float. The fit was determined to be valid if the modeled data had a Pearson coefficient greater than 0.8 when it was overlaid with the raw experimental data. The rate constant is a measure of the efficiency of the transporters as they take up the released dopamine. The value $\mathrm{k}$ can also be used to calculate $\mathrm{t}_{1 / 2}$, which is the measure of how long it takes half of the released dopamine to be uptaken.

The results of the modelling are shown in Figure 5. We found that there was no significant difference between any of the individual treatment groups. Thus, the data presented here point to uptake not being involved in the differences observed in $[\mathrm{DA}]_{\max }$ after treatment; however, more work needs to be done to both calculate the Michaelis-Menten kinetic parameters for zebrafish as well as address the effects of diffusion, which is not accounted for by this current method.

In summary, we have found that treatment of zebrafish with carboplatin, administered through water and food, impaired dopamine release in ex vivo whole brain preparations. However, neither treatment affected uptake. To our knowledge, this is the first published study that uses zebrafish as a model to examine the effects of cancer chemotherapeutics on neurotransmitter release. Moving forward, it is important to determine how chemotherapy treatment with carboplatin, as well as other chemotherapy agents, affect cognitive function in zebrafish. However, this work represents an important first step in the identification of neurochemical alterations by chemotherapy-treatment in zebrafish.

\section{Experimental Section}

\section{Drugs}

Pharmaceutical grade carboplatin, $10 \mathrm{mg} / \mathrm{mL}$ (CD11650AA, Hospira, Lake Forest, IL, USA) and $0.9 \%$ sterile saline (Nova-Tech Inc, Grand Island, NE, USA) solutions were used. Dopamine was purchased from Sigma-Aldrich (St. Louis, MO, USA). Aqueous solutions were prepared with purified (18.2 M $\Omega$ ) water. A modified artificial cerebrospinal fluid 
(aCSF) for zebrafish whole brain preparations consisted of $131 \mathrm{mM} \mathrm{NaCl}, 2 \mathrm{mM} \mathrm{KCl}, 1.25$ $\mathrm{mM} \mathrm{KH}_{2} \mathrm{PO}_{4}, 20 \mathrm{mM} \mathrm{NaHCO}_{3}, 2 \mathrm{mM} \mathrm{MgSO}_{4}, 10 \mathrm{mM}$ glucose, $2.5 \mathrm{mM} \mathrm{CaCl}_{2} \cdot \mathrm{H}_{2} \mathrm{O}$, and 10mM HEPES, and the $\mathrm{pH}$ was adjusted to 7.4.

\section{Brain preparation}

All animal procedures were approved by the University of Kansas Institutional Animal Care and Use Committee. Wild-type adult zebrafish, originally purchased from Carolina Biological Supply (Burlington, NC) or AquariumFish.net (San Diego, CA), were bred and housed 20 fish per 2L tank in the University of Kansas Synthetic Chemical Biology Core (SCBC). Zebrafish were maintained on a light dark cycle with a 16 hour light phase and an 8 hour dark phase. The temperature of the recirculating water system was maintained at $26{ }^{\circ} \mathrm{C}$.

Whole brains were harvested as previously described ${ }^{[5 a]}$. Briefly, for a given recording session, a zebrafish was euthanized by hypothermic shock and decapitated using a $0.009^{\prime \prime}$ single edge razor blade (VWR Corporates, Radnor, PA, USA). The head was transferred to a prepared dissection pad made of $2 \%$ agarose (BioReagent graded agarose, Sigma-Aldrich, St. Louis, MO, USA) in a $100 \mathrm{~mm} \times 15 \mathrm{~mm}$ petri dish (ThermoFisher Scientific, Waltham, MA, USA). The petri dish was filled with oxygenated (95\% O2/5\% CO2) ice-cold modified artificial cerebral spinal fluid. The head was then immobilized by pinning it to the agar with a syringe needle. The skull of the zebrafish was carefully removed using forceps, and the brain was removed with a pulled capillary and transferred to the recording chamber, which was perfused with oxygenated-modified aCSF kept at a physiological temperature of $28^{\circ} \mathrm{C}$.

\section{Chemotherapy treatment}

For chemotherapy exposure through the habitat water, fish were housed in $1 \mathrm{~L}$ of water to which pharmaceutical grade carboplatin in $0.9 \%$ saline $(10 \mathrm{mg} / \mathrm{mL})$ was added so that the final concentration of carboplatin was $100 \mu \mathrm{M}$. Control fish were housed in $1 \mathrm{~L}$ of water treated with an equal volume of $0.9 \%$ sterile biological saline. The fish were exposed continuously for 1, 4 or 7 days. Fresh solutions were made every 48 hours.

The oral treatment procedure consisted of soaking 1 gram of thawed, strained brine shrimp (San Francisco Bay Brand INC, Newark, CA, USA) for 30 minutes in $2 \mathrm{~mL}$ of carboplatin $(10 \mathrm{mg} / \mathrm{mL})$ or saline. A total weight $0.25 \mathrm{~g}$ of this shrimp was then added to a $1 \mathrm{~L}$ tank of the aquarium system water, and five fish were placed in this water and allowed to feed for a three-minute period. Fish were then removed from the feeding tank and placed in their home tank. This treatment was done once per day for 1,4 , or 7 days, after which the fish were sacrificed and brains analyzed.

\section{Estimation of carboplatin content in zebrafish food and body}

The brine shrimp were treated with carboplatin in a manner identical to how they were prepared for feeding. However, rather than placing the shrimp in the tank, we diluted them with enough water to bring the concentration of shrimp to $1 \mathrm{mg} / \mathrm{mL}$ and then homogenized the shrimp at room temperature $\left(\sim 23^{\circ} \mathrm{C}\right)$ by application of 15 strokes with a $1.5 \mathrm{~mL}$ Teflonglass tissue homogenizer (Vineland, NJ USA). The shrimp fragments were pelleted by 
centrifugation (5000 $\mathrm{g}$ for 15 minutes) and the supernatant was then analyzed for platinum content with graphite furnace atomic absorption (AA) spectroscopy.

To determine carboplatin retention in tissues, zebrafish were euthanized via hypothermic shock, dried, and weighed after 1 and 7 days of treatment. To facilitate homogenization, fish were cut into at least four pieces using a 0.009 " single edge razor blade (VWR Corporates, Radnor, PA, USA) and placed in a $2.0 \mathrm{~mL}$ microcentrifuge tube. Water was added $(1 \mathrm{~mL} / \mathrm{g}$ fish) and fish were homogenized at room temperature $\left(\sim 23^{\circ} \mathrm{C}\right)$ for 30 seconds with a D1000 handheld homogenizer (Benchmark Scientific, Edison, NJ, USA). Homogenates were pelleted by centrifugation (5000 $\mathrm{g}$ for 30 minutes) and the supernatants were analyzed with graphite furnace atomic absorption (AA) spectroscopy.

Samples from the food and body were analyzed in the following manner. A $10 \mu \mathrm{L}$ aliquot of each supernatant solution was injected into a graphite furnace (Analytical West, Corona, CA, USA). A hollow cathode Pt lamp (Photron LTD, Victoria, Australia) served as the light source. Each sample was atomized through the following heating cycle: 30 seconds of $125^{\circ} \mathrm{C}, 20$-second ramp to $1500^{\circ} \mathrm{C}, 30$-second hold at $1500^{\circ} \mathrm{C}$, and 8 seconds of $2700^{\circ} \mathrm{C}$. The peak height of the sample's absorbance at $266 \mathrm{~nm}$ was measured. The concentration of carboplatin was determined by comparison against an external calibration curve, which was prepared by injecting $10 \mu \mathrm{L}$ of $0,0.5,1.0,1.5,2.0,2.5$, and $3.0 \mu \mathrm{g} / \mathrm{mL}$.

\section{Electrochemistry}

Cylindrical carbon fiber microelectrodes were fabricated as previously described with minor modifications ${ }^{[14]}$. Briefly, a $7 \mu \mathrm{m}$ diameter carbon fiber (Goodfellow Cambridge LTD, Huntingdon, UK) was aspirated into glass capillary tubes (1.2 mm D.D and $0.68 \mathrm{~mm} \mathrm{I.D,} 4$ in long; A-M System Inc, Carlsborg, WA, USA). Loaded capillaries were then pulled using a PE-22 heated coil puller (Narishige Int. USA, East Meadow, NY, USA). Pulled carbon fibers were trimmed with a scalpel to a length of 50 to $70 \mu \mathrm{m}$ from the pulled glass tip. To seal the carbon fiber, electrodes were dipped into epoxy resin (EPON resin 815C and EPIKURE 3234 curing agent, Miller-Stephenson, Danbury, CT, USA) and cured at $100^{\circ} \mathrm{C}$ for 1 hour.

Electrochemical measurements were collected and analyzed using an electrochemical workstation consisting of a Dagan Chem-Clamp potentiostat (Dagan, Minneapolis, MN, USA), modified to allow gain settings down to $200 \mathrm{nA} / \mathrm{V}$, a personal computer with TarHeel CV software (provided by R.M. Wightman and M.L.A.V. Heien, University of North Carolina, Chapel Hill, NC, USA), a breakout box, and two National Instruments computer interface cards, PCI 6052 and PCI 6711 (National Instruments, Austin, TX, USA).

During a typical recording session, the brain was allowed to equilibrate in the perfusion chamber for a period of one hour. A carbon-fiber microelectrode and two stimulus electrodes (A-M Systems Inc., Carlsberg, WA, USA) were micromanipulated into a whole zebrafish brain as previously discussed ${ }^{[5 a]}$. The carbon-fiber microelectrode was positioned $50-100$ $\mu \mathrm{m}$ laterally from the medial olfactory tract (MOT) and inserted about $280-300 \mu \mathrm{m}$ deep. The stimulus electrodes were placed at the center of ventral telencephalon and inserted about $100 \mu \mathrm{m}$ into the brain so that the carbon-fiber microelectrode was positioned between stimulus electrodes. 
To evoke dopamine release, a stimulation train of 35 electrical pulses ( $350 \mu \mathrm{A}$ stimulating current, $4 \mathrm{~ms}$ of total duration, frequency of $60 \mathrm{~Hz}$ ) was applied. Evoked dopamine release was measured using a triangular waveform of $-0.4 \mathrm{~V}$ to $+1.3 \mathrm{~V}$ to $-0.4 \mathrm{~V}$ applied at a scan rate of $400 \mathrm{~V} / \mathrm{s}$ and an update frequency of $10 \mathrm{~Hz}$. After stimulation and dopamine detection, the brain was allowed a 10 minute resting period before the next stimulation event was applied. Evoked dopamine released was measured from either treated fish brain or control fish brain for 1 hour. Electrodes were pre-calibrated and post-calibrated against standard dopamine solutions. The average of the pre- and post-calibration was used to convert measured current in the brain to dopamine concentration.

\section{Data analysis and statistics}

All numerical values are represented as mean \pm standard error of the mean (SEM). For all analyses, $n$ was equal to the number of zebrafish brains used. GraphPad Prism 6 (GraphPad Software Inc, La Jolla, CA, USA) was used to conduct statistical calculations and to present data. The modelling was achieved by analyzing the raw data, deconvoluted by baseline subtraction, to determine the point of maximum dopamine signal after stimulation and the point where that signal had decayed by $80 \%$. This decay curve was then fit with the $1^{\text {st }}$ order exponential decay equation $A_{t}=A_{\max } e^{-k t}$. $A_{\max }$ is held constant at the experimentally determined value and $\mathrm{k}$, the $1^{\text {st }}$ order rate constant, is allowed to float. The accuracy of the fit is determined by using a Pearson coefficient with a cut off of $R \geq 0.8$ being used. Once this $\mathrm{k}$ is determined, the half-life of the decay was then calculated using the equation $\mathrm{t}_{1 / 2}=$ 0.6932/k. Data analysis and curve fitting was done using GraphPad Prism 6 (GraphPad Software Inc, La Jolla, CA, USA).

\section{Acknowledgments}

Funding was provided by the National Institutes of Health through the KU Center for Molecular Analysis of Disease Pathways (CMADP), grant number P20 GM103638 (MAJ) and an Exploratory/Developmental Research Grant Award 1R21NS077485 (MAJ). Additional support was provided by the Synthetic Chemical Biology Core facility of the CMADP. Co-author CSS was supported by NSF Research Experiences for Undergraduates (REU) grant CHE-1560279. Support was also provided by the University of Kansas.

\section{References}

1. Raffa RB, Duong PV, Finney J, Garber DA, Lam LM, Mathew SS, Patel NN, Plaskett KC, Shah M, Weng HFJ. J Clin Pharm Ther. 2006; 31:129-138. [PubMed: 16635046]

2. a Scheibel RS, Valentine AD, O’Brien S, Meyers CA. J Neuropsychiatry Clin Neurosci. 2004; 16:185-191. [PubMed: 15260370] b Meyers CA, Albitar M, Estey E. Cancer (Hoboken, NJ, U S). 2005; 104:788-793.c Ahles TA, Saykin AJ. Nature Reviews Cancer. 2007; 7:192-201. [PubMed: 17318212]

3. Kaplan SV, Limbocker RA, Gehringer RC, Divis JL, Osterhaus GL, Newby MD, Sofis MJ, Jarmolowicz DP, Newman BD, Mathews TA, Johnson MA. ACS Chem Neurosci. 2016; 7:689-699. [PubMed: 27145395]

4. a Eisenberger M, Hornedo J, Silva H, Donehower R, Spaulding M, Van Echo D. J Clin Oncol. 1986; 4:1506-1509. [PubMed: 3531424] b Kavanagh JJ, Nicaise C. Semin Oncol. 1989; 16:45-48.c Wagstaff AJ, Ward A, Benfield P, Heel RC. Drugs. 1989; 37:162-190. [PubMed: 2649354]

5. a Shin M, Field TM, Stucky CS, Furgurson MN, Johnson MA. ACS Chem Neurosci. 2017b Jones LJ, McCutcheon JE, Young AM, Norton WH. Front Behav Neurosci. 2015; 9:246. [PubMed: 26441575] 
6. Dai YJ, Jia YF, Chen N, Bian WP, Li QK, Ma YB, Chen YL, Pei DS. Environ Toxicol Chem. 2014; 33:11-17. [PubMed: 24307630]

7. Hinsch K, Zupanc GKH. Neuroscience. 2007; 146:679-696. [PubMed: 17395385]

8. a Naslund J. PeerJ. 2014; 2:e586. [PubMed: 25279266] b Ullmann JF, Cowin G, Kurniawan ND, Collin SP. NeuroImage. 2010; 51:76-82. [PubMed: 20139016]

9. Bambino K, Chu J. Curr Top Dev Biol. 2017; 124:331-367. [PubMed: 28335863]

10. Dallinger R, Prosi F, Segner H, Back H. Oecologia. 1987; 73:91-98. [PubMed: 28311410]

11. Heath, AG. Water pollution and fish physiology. CRC press; 1995.

12. Binks SP, Dobrota M. Biochemical pharmacology. 1990; 40:1329-1336. [PubMed: 2206139]

13. a Aluise CD, Sultana R, Tangpong J, Vore M, St Clair D, Moscow JA, Butterfield DA. Advances in experimental medicine and biology. 2010; 678:147-156. [PubMed: 20738017] b Butterfield DA. Free Radic Biol Med. 2014; 74:157-174. [PubMed: 24996204]

14. a Johnson MA, Rajan V, Miller CE, Wightman RM. J Neurochem. 2006; 97:737-746. [PubMed: 16573654] b Ortiz AN, Kurth BJ, Osterhaus GL, Johnson MA. J Neurochem. 2010; 112:755-761. [PubMed: 19929911] 

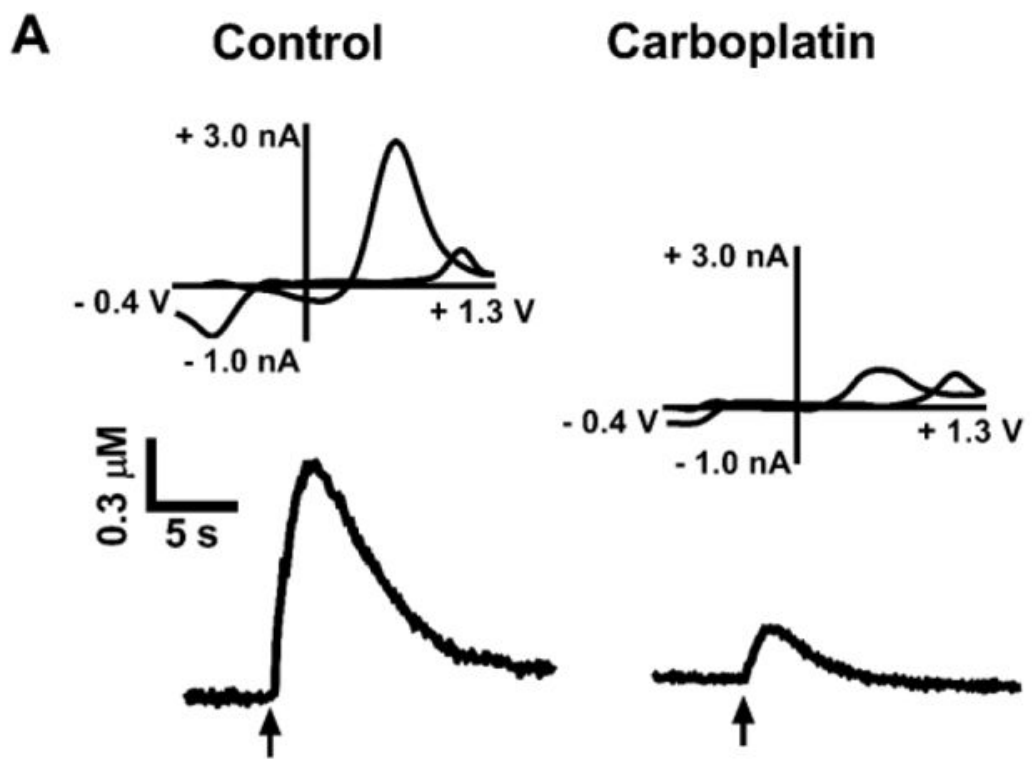

B

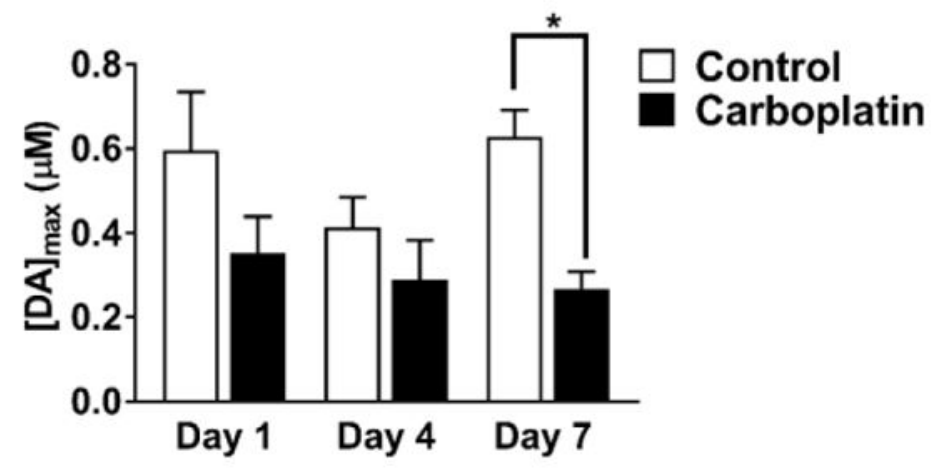

Figure 1.

Carboplatin administration though water. (A) Representative data for the control group and 7 days after $100 \mu \mathrm{M}$ carboplatin treatment. The cyclic voltammograms in both cases indicate that the neurotransmitter measured is dopamine. Arrow denotes time of stimulation. (B) Pooled measurements show a significant overall drug effect ( $\mathrm{p}<0.05$, two-way ANOVA, $\mathrm{n}=$ 5 brains) and a significant difference in release between the control and 7 day treatment groups (Sidak's test $* \mathrm{p}<0.05, \mathrm{n}=5$ brains per group). 

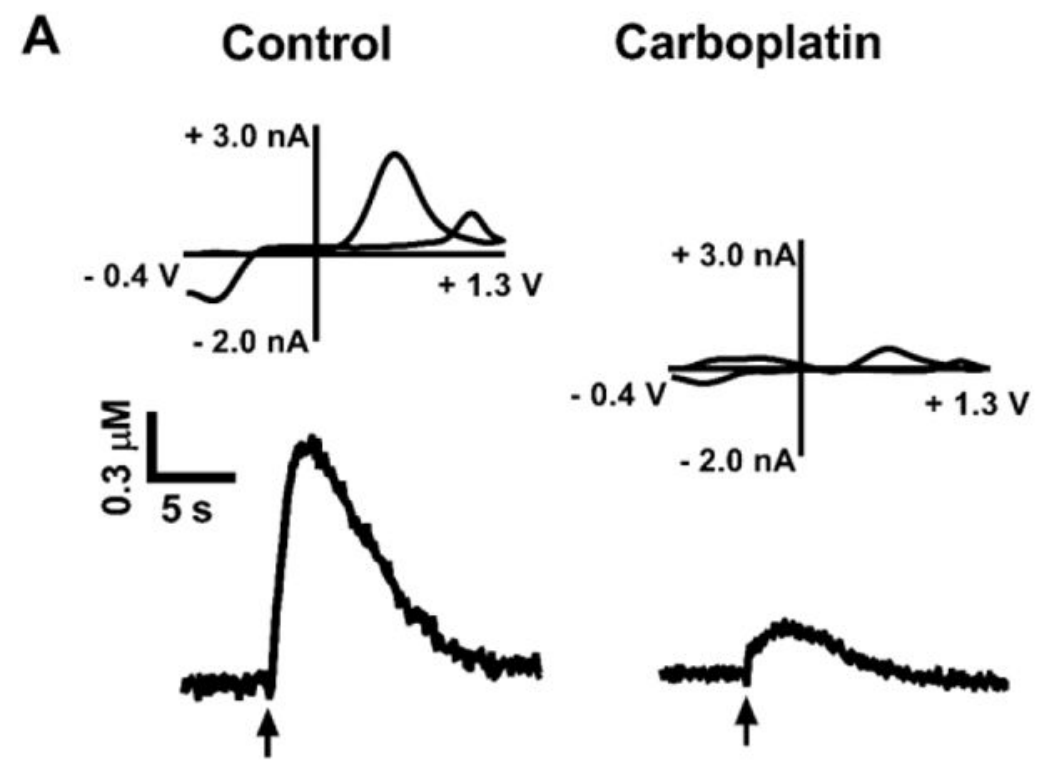

$-2.0 \mathrm{nA}$

B

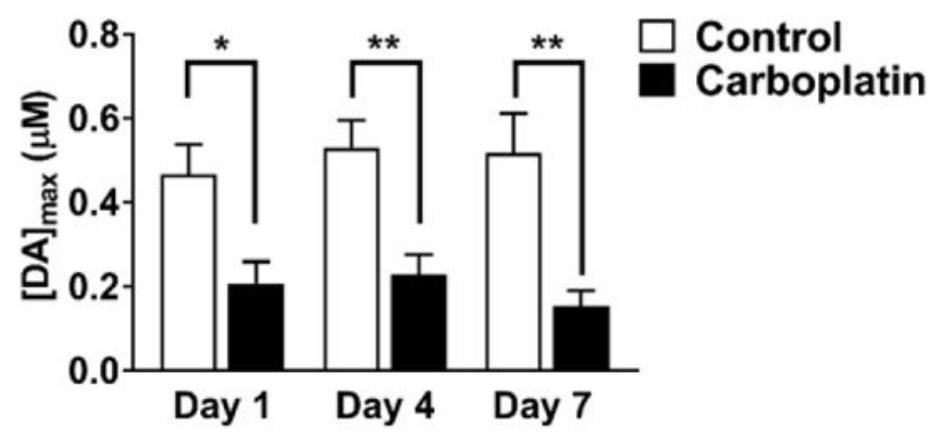

Figure 2.

Carboplatin administration through food. (A) Representative data showing an attenuation in dopamine release after 7 days of treatment. The cyclic voltammograms indicate the presence of dopamine. Arrow denotes time of stimulation. (B) The pooled data show a significant overall drug effect ( $p<0.0001$, two-way ANOVA) as well as a significant decrease in the dopamine release after 1,4 , and 7 days of treatment (Sidak's test $* \mathrm{p}<0.05$, ** $\mathrm{P}<0.01, \mathrm{n}=$ 5 brains per group). 

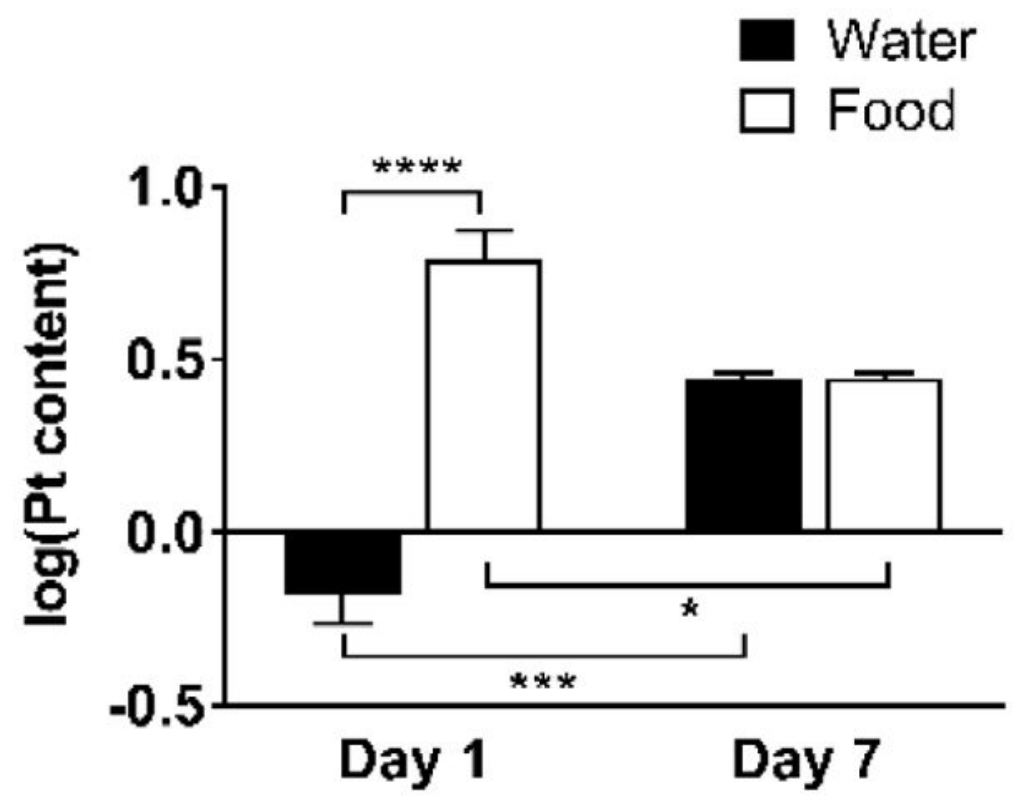

Figure 3.

Carboplatin content in whole zebrafish homogenates. Pt content in $\mathrm{mg} / \mathrm{kg}$ was $\log$ transformed for ease of comparison. There is a significant overall effect of route of administration ( $\mathrm{p}<0.0001$, two-way ANOVA, Sidak's test * $\mathrm{p}<0.05$, *** $\mathrm{p}<0.0005$, **** $\mathrm{p}$ $<0.0001, \mathrm{n}=3$ to 4 fish per group). 


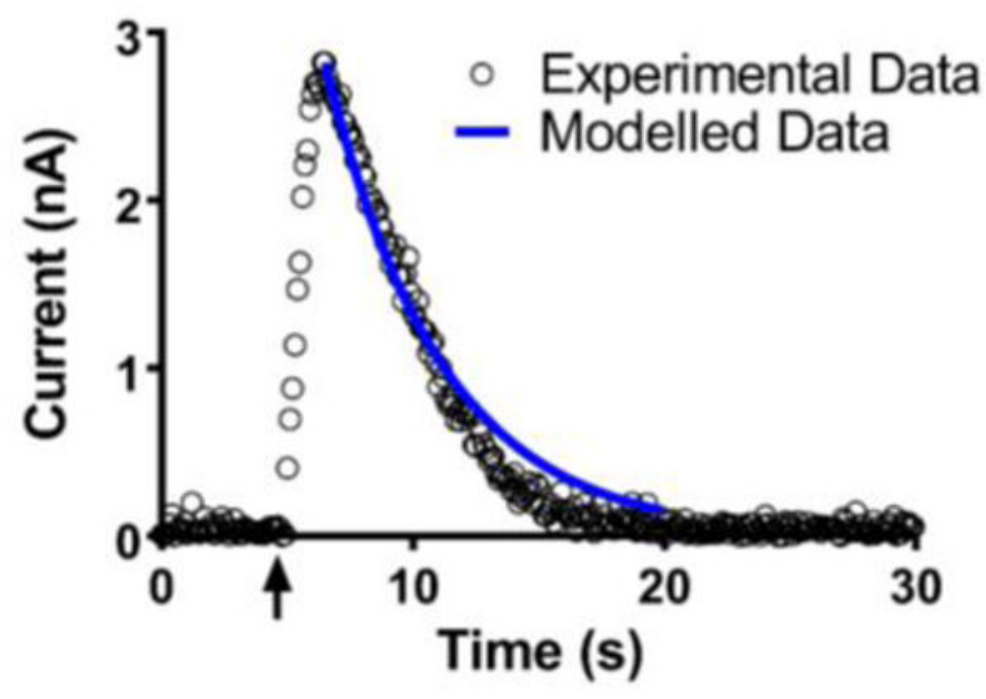

Figure 4.

Representative figure showing the raw experimental data (hollow circles) with the simulated data from the modeled parameters overlaid (blue line). Arrow denotes electrical stimulation. 

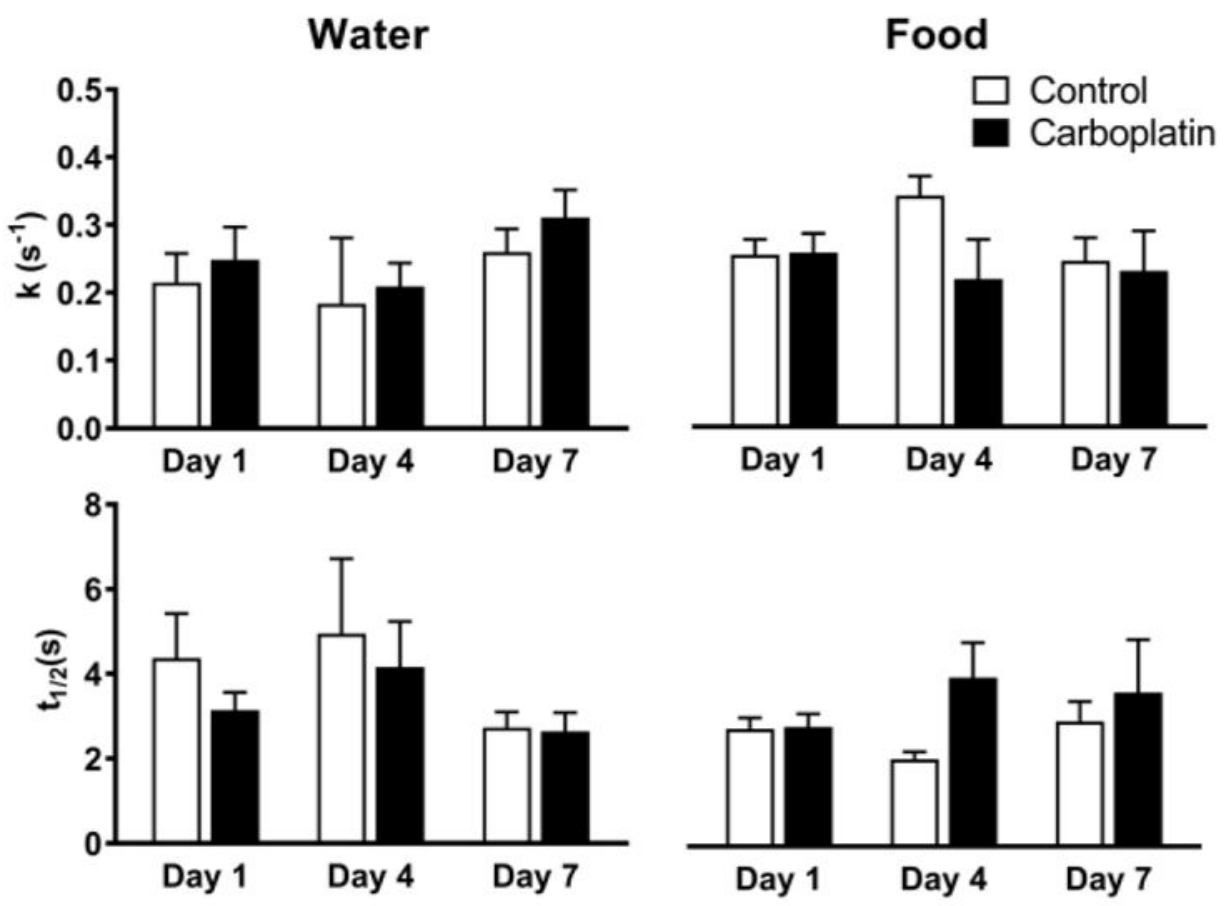

Figure 5.

The averages of the $1^{\text {st }}$ order rate constant, $\mathrm{k}$ (top panels) and half-life, $\mathrm{t}_{1 / 2}$ (bottom panels), in both water ('Water') and shrimp ('Food') treated zebrafish over the treatment time. There was no significant difference observed in either treatment pathway or treatment time (twoway ANOVA). 